\title{
Wireless Networks, Diversity and Space-Time Codes
}

\author{
Yindi Jing \\ Dept. of Electrical Engineering \\ California Institute of Technology \\ Pasadena, CA 91125 \\ e-mail: yindi@caltech.edu
}

\author{
Babak Hassibi ${ }^{\prime}$ \\ Dept. of Electrical Engineering \\ California Institute of Technology \\ Pasadena, CA 91125 \\ e-mail: hassibiecaltech.edu
}

\begin{abstract}
We apply the idea of space-time coding devised for multiple-antenna systems to the problem of communications over wircless relay networks. A two-stage protocol is used, where in one stage the transmitter sends information and in the other, the relay nodes encode their received signals into a "distributed" linear dispersion code, and then transmit the coded signals to the receiver. We show that for high SNR the proposed system has a diversity of order $\alpha_{0} \min \{T, R\}$, with $T$ the coherence interval, $R$ the number of relay nodes, and $\alpha_{0}$ the solution to the equation $\alpha+\frac{\log \alpha}{\log P}=1-\frac{\log \log P}{\log P}$, where $P$ is the total transmit power in the network. In particular, we show that the painwise error probability $(P E P)$ decays no slower than $\left(\frac{\log P}{P}\right)^{\min \{T, R\}}$. Thus, apart from the $\log P$ factor and assuming $T \geq R$, the system has the same diversity as a multiple-antenna system with $R$ transmit antennas and one receive antenna, which is the same as assuming that the $R$ relay nodes can fully cooperate and have full knowledge of the transmit signal. We further show that for a fixed total transmit power across the entire network, the optimal power allocation is for the transmitter to expend half the power and for the relays to collectively expend the other half. We also show that at low and high SNR, the coding gain is the same as that of multiple-antenna systems. However, at intermediate SNR, it can be quite different. We discuss some of the ramifications of using different space-time codes and finally verify our analysis through the simulation of randomly generated distributed space-time codes.
\end{abstract}

\section{INTRODUCTION}

It is known that multiple antennas can greatly increase the capacity and reliability of a wireless communication link in a fading environment using space-time codes $[1,2,3,4]$. Recently, with the increasing interests in ad hoc networks, researchers have been looking for methods to exploit spatial diversity using the antennas of different users in the network $[5,6,7,8,9]$. In [8], the authors exploit spatial diversity using the repetition and space-time algorithms. The mutual information and outage probability of the network are analyzed. However, in their model, the relay nodes need to decode their received signals. In [9], a network with a single relay under different protocois is analyzed and second order spatial diversity is achieved. In [10], the authors use space-time codes based on Hurwitz-Radon matrices and conjecture a diversity factor around $R / 2$ from their simulations. Also, the simulations in [11] show that the use of Khatri-Rao codes lowers the average bit error rate. In this paper, we consider a relay network with fading and apply a linear dispersion space-time code [12] among the relays. The problem we are interested in is: "Can we increase the reliability of a wireless network by using space-time codes among the relay nodes?"

A key feature of our work is that we do not require the relay nodes to decode. This has two main benefits: first, the operations at the relay nodes are considerably simplified, and second, we can avoid

\footnotetext{
I This work was supported in part by the National Science Foundation under grant no. CCR-0133818, by the office of Naval Research under grant no. N00014-02-1-0578, and by Caltech's Lee Center for Advanced Networking.
}

imposing bottlenecks on the rate by requiring some relay nodes to decode (see e.g., [13]).

The wireless relay network model we use is similar to those in $[14,15]$. In [14], the authors show that the capacity of the wireless relay network with $n$ nodes behaves like $\log n$. In [15], a power efficiency that behaves like $\sqrt{n}$ is obtained. Both results are based on the assumption that every relay node knows its local channels so that they can work coherently. Therefore, for the results of [14] and [15] to hold, the system should be synchronized at the carrier level. In this paper, we assume that the relay nodes do not know the channel information. All we need is a much more reasonable assumption that the system is synchronized at the symbol level.

First, we focuses on the basic results on cooperative diversity gain achieved using simple linear dispersion codes among the distributed relay nodes. Our work shows that using linear dispersion space-time codes among the relay nodes can achieve a diversity of $\min \{T, R\}\left(1-\frac{\log \log P}{\log P}\right)$. When $T \geq R$, the transmit diversity is linear in the number of relays (size of the network) and is a function of the average total transmit power. When $P$ is very large, the diversity is approximately $R$. The coding gain for very large $P(P \gg \log P)$ is $\operatorname{det}\left(S_{i}-S_{j}\right)^{*}\left(S_{i}-S_{j}\right)$, where $S_{i}$ and $S_{j}$ are codewords in the distributed space-time code. Therefore, at asymptotically high SNR, the same transmit diversity and coding gain are obtained as in the multiple-antenna case, which means that the system works as if the relays can fully cooperate and have full knowledge of the signal.

Then, we slightly improve the diversity gain achieved and prove the optimality of the result. We also consider a more general type of linear dispersion codes which includes Alamouti's scheme as a special case. The same diversity gains are achieved using this more general type of linear dispersion codes. However, the coding gain can be improved. We also simulate the performance of wireless relay networks using distributed space-time codes and compare with that of multiple-antenna systems using the same space-time codes.

\section{WIRELESS RELAY NETWORK}

Consider a wireless network with $R+2$ nodes which are placed randomly and independently according to some distribution. There is one transmit node and one receive node. All the other $R$ nodes work as relays. Every node has one antenna and antennas at relay nodes can be used for both transmission and reception. Denote the channel from the transmitter to the $i$ th relay as $f_{i}$, and the channel from the $i$ th relay to the receiver as $g_{i}$. Assume that $f_{i}$ and $g_{i}$ are independent complex Gaussian with zero-mean and unit-variance. If the fading coefficients $f_{i}$ and $g_{i}$ are known to relay $i$, it is proved in [14] and [15] that the capacity behaves like $\log R$ and a power efficiency that behaves like $\sqrt{R}$ can be obtained. However, these results rely on the assumption that the relay nodes know their local connections, which requires the system to be synchronized at the carrier level. In this paper, we make the much more practical assumption that the relay nodes are only coherent at the symbol level. In our relay network, we assume that the relay nodes know only the statistical distribution of the channels. However, we make the assumption that the receiver 
knows all the fading coefficients $f_{i}$ and $g_{i}$. Its knowledge of the channets can be obtained by sending training signals from the relays and the transmitter. Our main question is what gains can be obtained? There are two types of gains: improvement in the outage capacity and improvement in the PEP. In this paper, we focus on the latter.

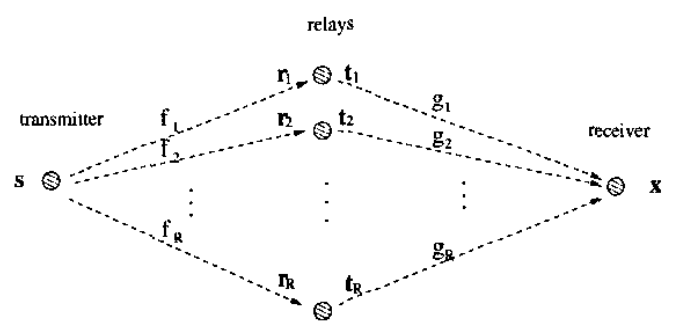

Figure 1: Wireless relay network

Assume that the transmitter wants to send the signal $\mathbf{s}=$ $\left[s_{1}, \cdots, s_{T}\right]^{t}$ in the codebook $\left\{s_{1}, \cdots, s_{L}\right\}$ to the receiver, where $L$ is the cardinality of the codebook. $s$ is normalized as

$$
E s^{*} s=1 .
$$

$\mathrm{s}^{t}$ and $\mathrm{s}^{*}$ indicates the transpose and conjugate transpose of $\mathrm{s}$, respectively. The transmission is accomplished by the following two-step strategy, which is also shown in Fig 1. From time 1 to $T$, the transmitter sends signals $\sqrt{P_{1} T} s_{1}, \cdots, \sqrt{P_{1} T} s_{T}$ to each relay. Based on (1), the average total transmit power of the $T$ transmissions is $P_{1} T$. The received signal at the $i$ th relay at time $\tau$ is denoted as $r_{i, \tau}$, which is corrupted by the noise $v_{i, \tau}$. From time $T+1$ to $2 T$, the $i$ th relay node transmits $t_{i, 1}, \cdots, t_{i, T}$ to the receiver based on its received signals. We denote the received signal at the receiver at time $\tau+T$ by $x_{\tau}$, and the noise at the receiver at time $\tau+T$ by $w_{\tau}$. Assume that the noises are complex Gaussian with zero-mean and unit-variance, that is, the distribution of $v_{i, \tau}, w_{\tau}$ are $\mathcal{C N}(0,1)$. Define

$\mathbf{v}_{i}=\left[\begin{array}{c}v_{i, 1} \\ \vdots \\ v_{i, T}\end{array}\right], \mathbf{r}_{i}=\left[\begin{array}{c}r_{i, 1} \\ \vdots \\ r_{i, T}\end{array}\right], \mathbf{t}_{i}=\left[\begin{array}{c}t_{i, 1} \\ \vdots \\ t_{i, T}\end{array}\right], \mathbf{w}=\left[\begin{array}{c}w_{1} \\ \vdots \\ w_{T}\end{array}\right]$, and $\mathbf{x}=\left[\begin{array}{c}x_{1} \\ \vdots \\ x_{T}\end{array}\right]$

If $f_{i}$ and $g_{i}$ keep constant for $T$ transmissions, clearly

$$
\mathbf{r}_{i}=\sqrt{P_{1} T} f_{i} \mathbf{s}+\mathbf{v}_{i}, \quad \text { and } \quad \mathbf{x}=\sum_{i=1}^{R} g_{i} \mathbf{t}_{i}+\mathbf{w}
$$

\section{Distributed SPACe-Time CODING}

From the above description, it is clear that if the transmission rate is sufficiently low, then all the relay nodes can decode the transmitted message. In this case, the relay nodes can act as a multiple-antenna system with $R$ transmit antennas and one receive antenna and therefore communications from the relay nodes to the receiver can achieve diversity $R$. This approach, however, will require a substantial reduction of the rate and we will not consider it. We will instead focus on the diversity achievable without requiring the relay nodes to decode. ${ }^{1}$

In this paper, we use the idea of the linear dispersion space-time code [12] for multiple-antenna systems by designing the transmitted

\footnotetext{
${ }^{\mathrm{I}} \mathrm{A}$ combination of requiring some relay nodes to decode and others to not, may also considered. However, in the interest of space, we shall not do so here.
}

signal at every relay node as a linear function of its received signal, that is,

$$
\mathbf{t}_{i}=\sqrt{P_{2} /\left(P_{1}+1\right)} A_{i} \mathbf{r}_{i},
$$

where $A_{i}$ is a $T \times T$ matrix. While within the framework of linear dispersion codes, the $T \times T$ matrices $A_{i}$ can be quite arbitrary (apart from a Frobenius norm constraint), to have a protocol that is equitable among different users and among different time instants, we shall henceforth assume that the $A_{i}$ are unitary. This also simplifies the analysis considerably. With the normalization in (2), the average transmit power at every relay node is $P_{2}$ per transmission.

The received signal can therefore be written as,

$$
\mathbf{x}=\sqrt{P_{1} P_{2} T /\left(P_{1}+1\right)} S H+W,
$$

where we have defined

$$
S=\left[\begin{array}{lll}
A_{1} \mathbf{s} & \cdots & A_{R} \mathbf{s}
\end{array}\right], \quad H=\left[\begin{array}{lll}
f_{1} g_{1} & \cdots f_{R} g_{R}
\end{array}\right]^{t},
$$

and

$$
W=\sqrt{P_{2} /\left(P_{1}+1\right)} \sum_{i=1}^{R} g_{i} A_{i} \mathbf{v}_{i}+\mathbf{w} .
$$

(3) shows that the $T \times R$ matrix $S$ works like the space-time code in multiple-antenna systems. We call it the distributed space-time code to emphasize that it has been generated in a distributed way by the relay nodes, without having access to $\mathrm{s} . H$, which is $R \times 1$, is the equivalent channel matrix and $W$, which is $T \times 1$, is the equivalent noise. $W$ is clearly influenced by the choice of the space-time code.

When both $f_{i}$ and $g_{i}$ are known to the receiver, it can be calculated that $\mathbf{x} \mid \mathbf{s}_{i}$ is Gaussian with mean $\sqrt{P_{1} P_{2} T /\left(P_{1}+1\right)} S_{i} H$ and variance $\left(1+P_{2} /\left(P_{1}+1\right) \sum_{i=1}^{R}\left|g_{i}\right|^{2}\right) I_{T}$. Therefore,

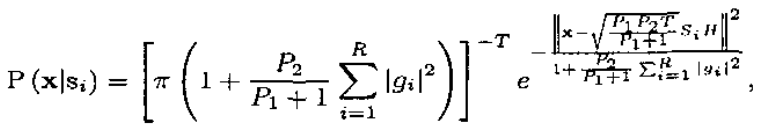

from which the ML decoding can be written as

$$
\arg \min _{\mathbf{s}_{i}}\left\|\mathbf{x}-\sqrt{P_{1} P_{2} T /\left(P_{1}+1\right)} S_{i} H\right\|^{2},
$$

where $\|\cdot\|$ indicates the Frobenius norm. Since $S_{i}$ is linear in $\mathbf{s}_{i}$, (4) is equivalent to the decoding of a linear system and sphere decoding can be used $[18,19]$.

Theurem 1 (Chernoff bound on the PEP). With the ML decoding in (4), the PEP, averaged over the channel coefficients, of mistaking $\mathbf{s}_{i}$ by $\mathbf{s}_{j}$ has the following Chemoff bound.

$$
P e \leq \underset{g_{i}}{\mathrm{E}} \operatorname{det}^{-1}\left[I_{R}+\frac{P_{1} P_{2} T}{4\left(1+P_{1}+P_{2} \sum_{i=1}^{R}\left|g_{i}\right|^{2}\right)} M G\right],
$$

where $\left.M=\left(S_{i}-S_{j}\right)^{*}\left(S_{i}-S_{j}\right), G=\operatorname{diag}\left\{\mid g_{1}\right\}^{2}, \cdots,\left|g_{R}\right|^{2}\right\}$, and det indicates the determinant.

We omit the proof due to the lack of space. ${ }^{3}$ Let's compare (5) with the Chernoff bound on the PEP of a multi-antenna system with

\footnotetext{
${ }^{2}$ Note that the conjugate of $r_{i}, \overline{r_{i}}$, does not appear in (2). The case with $\overline{r_{i}}$ will be discussed later in Section VII

${ }^{3}$ For proofs of all the theorems and Corollary, see [16] and [17].
} 
$R$ transmit antennas and one receive antenna (the receiver knows the channel) $[4,20]$ :

$$
P e \leq \operatorname{det}^{-1}\left[I_{R}+\frac{P T}{4 R} M\right] .
$$

The difference is that now we need to do the expectations over $g_{i}$. Before that, similar to the multiple-antenna case, the "full diversity" condition can be obtained from (5). It is easy to see that if $S_{i}-S_{j}$ drops rank, the upper bound in (5) increases. Therefore, the Chernoff bound is minimized when $S_{i}-S_{j}$ is full-rank, or equivalently, $\operatorname{det} M \neq 0$.

\section{POWER Allocation}

Now let's discuss the optimum power allocation between the transmitter and relays that minimizes the PEP. Because of the expectations over $g_{i}$, this is easier said than done. We shall therefore do this approximately. Note that $g=\sum_{i=1}^{R}\left|g_{i}\right|^{2}$ has the Gamma distribution whose mean and variance are both $R$. Thus, by the law of large numbers, almost surely $\frac{1}{R} g \rightarrow 1$ when $R \rightarrow \infty$. It is therefore reasonable to approximate $g$ by its mean, i.e., $g \approx R$, especially for large $R$. Therefore, (5) becomes

$$
P e \lesssim \underset{g_{i}}{\mathrm{E}} \operatorname{det}^{-1}\left[I_{R}+\frac{P_{1} P_{2} T}{4\left(1+P_{1}+P_{2} R\right)} M G\right] .
$$

We can see that the upper bound in (6) is minimized when $P_{1} P_{2} T / 4\left(1+P_{1}+P_{2} R\right)$ is maximized.

Assume that the total power consumed is $P T$ for transmissions of $T$ symbols. Since the powers used at the transmitter and every relay are $P_{1}$ and $P_{2}$ for each transmission, $P=P_{1}+R P_{2}$. Therefore, for $P \gg 1$,

$$
\frac{P_{1} P_{2} T}{4\left(1+P_{1}+P_{2} R\right)} \leq \frac{P^{2} T}{16 R(1+P)} \approx \frac{P T}{16 R}
$$

with equality when

$$
P_{1}=\frac{P}{2} \quad \text { and } \quad P_{2}=\frac{P}{2 R}
$$

Therefore, the optimum power allocation is such that the transmitter uses half the total power and the relays share the other half fairly. For large $R$, the relays spend only a very small amount of power to help the transmitter. With this optimum power allocation, for $P \gg 1,(5)$ becomes

$$
P e \lesssim \underset{g_{i}}{\mathrm{E}} \operatorname{det}^{-1}\left[I_{R}+\frac{P T}{8\left(R+\sum_{i=1}^{R}\left|g_{i}\right|^{2}\right)} M G\right] .
$$

\section{BASIC Diversity Results}

As mentioned earlier, to obtain the diversity we need to compute the expectation in (5). We shall do this rigorously later. However, since the calculations are detailed and give little insight, we begin by giving a simple approximate derivation which leads to the same diversity result. As discussed in the previous section, when $R$ is large, $g \approx R$ with high probability. We use this approximation first to simplify the derivation. We upper bound the PEP using the minimum nonzero singular value of $M$, which is denoted as $\sigma^{2}$. From (5) and (7),

$$
\begin{aligned}
& \underset{P e}{\lesssim} \underset{g_{i}}{\mathrm{E}} \operatorname{det}^{-1}\left[I_{R}+\frac{P T \sigma^{2}}{16 R} \operatorname{diag}\left\{I_{\text {rank } M, 0\} C]}\right]\right. \\
= & \underset{g_{i}}{\mathrm{E}} \prod_{i=1}^{\text {rank } M}\left(1+\frac{P T \sigma^{2}}{16 R}\left|g_{i}\right|^{2}\right)^{-1} \\
= & {\left[\int_{0}^{\infty}\left(1+\frac{P T \sigma^{2}}{16 R} x\right)^{-1} e^{-x} d x\right]^{\text {rank } M} } \\
= & \left(\frac{P T \sigma^{2}}{16 R}\right)^{-\operatorname{rank} M}\left[-e^{-\frac{16 R}{P^{\prime} T \sigma^{2}}} \mathbf{E i}\left(-\frac{16 R}{P T \sigma^{2}}\right)\right]^{\operatorname{rank} M}
\end{aligned}
$$

where $\operatorname{Ei}(\chi)=\int_{-\infty}^{\chi} \frac{e^{t}}{t} d t, \quad \chi<0$ is the exponential integral function [22]. Also,

$$
\operatorname{Ei}(\chi)=c+\log (-\chi)+\sum_{k=1}^{\infty} \frac{(-1)^{k} \chi^{k}}{k \cdot k !}
$$

with $c$ the Euler constant and $\log$ the natural logarithm. For $P \gg 1$, $e^{-\frac{16 R}{P T \sigma_{\min }^{2}}} \approx 1$ and $-\mathbf{E i}\left(-\frac{16 R}{P T \sigma_{m \text { min }}^{2}}\right) \approx \log P$. Therefore,

$$
\begin{aligned}
P e & \lesssim\left(16 R / T \sigma_{\min }^{2}\right)^{\operatorname{rank} M}(\log P / P)^{\operatorname{rank} M} \\
& =\left(16 R / T \sigma_{\min }^{2}\right)^{\operatorname{rank} M} P^{\operatorname{rank} M\left(1-\frac{\mathrm{kgg} \log P}{\operatorname{lng} P}\right)}
\end{aligned}
$$

If $M$ is full rank, diversity, $\min \{T, R\}\left(1-\frac{\log \log P}{\log P}\right)$, is obtained. Therefore, similar to the multiple-antenna case, there is no point in having more relays than the coherence interval. Thus, we will henceforth assume $T \geq R$. The transmit diversity is therefore $R\left(1-\frac{\log \log P}{\log P}\right)$. (10) also shows that the PEP is smaller for bigger coherence interval $T$. A rigorous result is given in the following theorem.

Theorem 2. Design the transmit signal at the ith relay node as in (2) and use the power allocation in (8). For full diversity of the space. time code, assume $T \geq R$. For any $x>0$,

$$
\begin{aligned}
P e \leq & \sum_{k=0}^{R}\left(\frac{8}{P T}\right)^{k} \sum_{1 \leq i_{1}<\cdots<i_{k} \leq R} \operatorname{det}^{-1}[M]_{i_{1}, \cdots, i_{k}} \\
& \left(1-e^{-x}\right)^{R-k} \sum_{j=0}^{k} B_{R+(R-k) x, x}(j, k)[-\mathbf{E i}(-x)]^{k-j},
\end{aligned}
$$

where

$$
\begin{aligned}
B_{A, x}(j, k)= & \left(\begin{array}{c}
k \\
j
\end{array}\right) \sum_{i_{1}=1}^{k} \sum_{i_{2}=1}^{k-i_{1}} \cdots \sum_{i_{j}=1}^{k-i_{1}+\cdots-i_{j-1}} \\
& \left(\begin{array}{c}
k \\
i_{1}
\end{array}\right) \cdots\left(\begin{array}{c}
k-i_{1}-\cdots-i_{j-1} \\
i_{j}
\end{array}\right) \\
& \Gamma\left(i_{1}, x\right) \cdots \Gamma\left(i_{j}, x\right) A^{k-i_{1}-\cdots-i_{j}}
\end{aligned}
$$

and $[M]_{i_{1}, \cdots, i_{k}}$ is the $k \times k$ matrix composed by the $i_{1}, \cdots, i_{k}$ th rows and columns of $M$.

Idea of the proof: To upper bound the $R$ integrals in (5), we first break every integral into two parts: the integration from 0 to $x$ and the integration from $x$ to $\infty$, and then upper bound every one of the resulting $2^{R}$ terms. 
Corollary 1. If $\log P \gg 1$,

$$
\begin{aligned}
P e \lesssim \frac{1}{P^{R}} \sum_{k=0}^{R}\left(\frac{8}{T}\right)^{k} \sum_{\substack{1 \leq i_{1}<\cdots<i_{k} \leq R\\
}} \operatorname{det}^{-1}[M]_{i_{1}, \cdots, i_{k}} \\
\\
\sum_{l=0}^{k} B_{R, 0}(k-l, k) \log ^{l} P .
\end{aligned}
$$

If $R \gg 1$

$$
P e \lesssim \frac{1}{P^{R}} \sum_{k=0}^{R}\left(\frac{8 R}{T}\right)_{1 \leq i_{1}<\cdots<i_{k} \leq R}^{k} \operatorname{det}^{-1}[M]_{i_{1}, \cdots, i_{k}} \log ^{k} P .
$$

Proof: Choose $x=1 / P$. (11) is obtained by ignoring higher order terms of $1 / P$. When $R \gg 1, B_{R}(0, k)>>B_{R}(l, k)$ for all $l>0$ since $B_{R}(0, k)=R^{k}$ is the term with the highest order of $R$. Therefore, (12) is obtained from (11).

The $k=l=R$ term in (11),

$$
\begin{aligned}
& \operatorname{det}^{-1} M\left(\frac{8 R \log P}{T P}\right)^{R} \\
= & \operatorname{det}^{-1} M\left(\frac{8 R}{T}\right)^{R} P^{-R\left(1-\frac{\log \log P}{\log P}\right)},
\end{aligned}
$$

has the highest order of $P$. Therefore, diversity, $R\left(1-\frac{\log \log P}{\log P}\right)$, is obtained.

In multiple-antenna systems with $R$ transmit antennas and one receive antenna, at high transmit power, the PEP has the upper bound $\frac{1}{P^{R}}$ det $^{-1} M\left(\frac{4 R}{T}\right)^{R}$. Comparing this with the highest order term given in (13), we can see the relay network has a performance that is

$$
\left(3+10 \log _{10} \log P\right) d B
$$

worse, where $\log _{10}$ indicates the base 10 logarithm. This analysis is verified by simulations in Section VIII.

Corollary 1 also gives the coding gain for networks with large number of relay nodes. When $P$ is very $\operatorname{large}(\log P \gg 1)$, the dominant term in (12) is (13). The coding gain is therefore $\operatorname{det}^{-1} M$, which is the same as the multiple antenna case. When $P$ is not very large, the second term (the $k=R-1$ term ) in (12) cannot be ignored and even the $k=3,4, \cdots$ terms have non-negligible contributions. Therefore, we want not only $\operatorname{det} M$ to be large but also $\operatorname{det}[M]_{i_{1}, \cdots, i_{k}}$ to be large for all $0 \leq k \leq R, 1 \leq i_{1}<\cdots<i_{k} \leq$ $R$. Note that

$[M]_{i_{1}, \cdots, i_{k}}=\left(\left[S_{i}\right]_{i_{1}, \cdots, i_{k}}-\left[S_{j}\right]_{i_{1}, \cdots, i_{k}}\right)^{*}\left(\left[S_{i}\right]_{i_{1}, \cdots, i_{k}}-\left[S_{j}\right]_{i_{1}}, \cdots, i_{k}\right)$, where $\left[S_{i}\right]_{i_{1}}, \cdots, i_{k}=\left(A_{i_{1}} \mathbf{s}_{i}, \cdots, A_{i_{k}} \mathbf{s}_{i}\right)$ is the space-time code when only the $i_{1}, \cdots, i_{k}$ th relay nodes are working. To have a good performance for not very large transmit power, both Theorem 2 and Corollary 1 indicate that the distributed space-time code should have the property that it is "scale-free" in the sense that it is still a good distributed space-time code when some of the relays are not working.

If $P \ll 1$, with the approximation $\sum_{i=1}^{R}\left|g_{i}\right|^{2} \approx R$ and the power allocation given in (8), (9) can be calculated to be

$$
P e \lesssim 1-\frac{P^{2} T}{16 R} \operatorname{tr} M+o\left(P^{2}\right)
$$

where $\mathrm{tr}$ indicates the trace. Similar to the multiple-antenna case, the coding gain is $\operatorname{tr} M$. The design criterion is to maximize $\operatorname{tr} M$.

\section{IMPROVEMENT IN DIVERSITY}

In Corollary 1 , we have chosen $x=1 / P$, which turns out to not give the tightest bound. In fact, we can improve the diversity slightly.

Theorem 3. The best diversity that can be obtained using the distributed space-time code in (2) is $\alpha_{0} R$, where $\alpha_{0}$ is the solution of

$$
\alpha+\frac{\log \alpha}{\log P}=1-\frac{\log \log P}{\log P} .
$$

If $P \gg \log P$ and $R \gg 1$,

$P e \lesssim \sum_{k=0}^{R}\left(\frac{8 R}{T}\right)^{k} \sum_{1 \leq i_{1}<\cdots<i_{k} \leq R} \operatorname{det}^{-1}[M]_{i_{1}, \cdots, i_{k}} P^{-\alpha_{0} R}$.

There is no closed-form solution for equation (15). The following theorem gives a region of $\alpha_{0}$.

Theorem 4. For $P>e$,

$1-\frac{\log \log P}{\log P}<\alpha_{0}<1-\frac{\log \log P}{\log P}+\frac{\log \log P}{\log P(\log P-\log \log P)}$.

In terms of diversity, Theorem 4 indicates that the PEP Chernoff bound of the distributed space-time codes decreases faster than $\left(\frac{\log P}{P}\right)^{R}$ and slower than $\left(\frac{(\log P)^{1-\frac{10}{6 g} P-\log \log P}}{P}\right)^{R}$. When $P$ is large $(P \gg \log P), 1-\frac{\log \log P}{\log P}$ is a very accurate approximation of $\alpha_{0}$. The improvement in diversity is small.

Now let's compare (16) with (12). A slightly better transmit diversity is obtained as discussed above. However, the coding gain in (16) is smaller. To compare the two, we assume that the singular values of $M$ take their maximum value, $\sqrt{2}$, and $R=T$. The coding gain of (16) can be calculated to be $5^{-R}$. The coding gain of $(12)$ is $4^{-R}$. The upper bound in (12) is $0.97 \mathrm{~dB}$ better according to coding gain. Therefore, when $P$ is extremely large, the new upper bound is tighter than the previous one since it has a larger diversity. Otherwise, the previous bound is tighter since it has a larger coding gain.

\section{ViI. A More General Type of Distributed SPACE-TIME CODES}

Note that $\overline{r_{i}}$ does not appear in (2). In this section, we work on a more general type of distributed linear dispersion space-time codes [12] by designing the transmitted signal at the $i$ th relay node as.

$$
\mathbf{t}_{i}=\sqrt{\frac{P_{2}}{P_{1}+1}}\left(A_{i} \mathbf{r}_{i}+B_{i} \overline{\mathbf{r}_{i}}\right), \quad i=1,2, \cdots, R,
$$

where $A_{i}, B_{i}$ are $T \times T$ real matrices. Similar as before, we assume that $A_{i}+B_{i}$ and $A_{i}-B_{i}$ are orthogonal. By separating the real and imaginary parts, we can write (17) equivalently as

$$
\left[\begin{array}{c}
t_{i, R e} \\
\mathbf{t}_{i, I m}
\end{array}\right]=\sqrt{\frac{P_{2}}{P_{i}+1}}\left[\begin{array}{cc}
A_{i}+B_{i} & 0 \\
0 & A_{i}-B_{i}
\end{array}\right]\left[\begin{array}{c}
\mathbf{r}_{i, R e} \\
\mathbf{r}_{i, I m}
\end{array}\right],
$$

where $t_{i, R e}$ and $t_{i, I m}$ indicate the real and imaginary parts of $t_{i}$. The expected total transmit power at the $i$ th relay can therefore be calculated to be $P_{2} T$.

For any $T \times 1$ complex vector $\mathbf{x}$, define the $2 T \times 1$ real vector $\hat{\mathbf{x}}=\left[\begin{array}{l}\mathbf{x}_{R e} \\ \mathbf{x}_{I m}\end{array}\right]$. The following system equation can be derived.

$$
\hat{\mathbf{x}}=\sqrt{\frac{P_{1} P_{2} T}{P_{1}+1}} \mathcal{H} \hat{s}+\mathcal{W},
$$


where the $2 T \times 2 T$ matrix,

$$
\begin{array}{r}
\mathcal{H}=\sum_{i=1}^{R}\left[\begin{array}{cc}
g_{i, R e} I_{T} & -g_{i, I m} I_{T} \\
g_{i, I m} I_{T} & g_{i, R e} I_{T}
\end{array}\right]\left[\begin{array}{cc}
A_{i}+B_{i} & 0 \\
0 & A_{i}-B_{i}
\end{array}\right] \\
{\left[\begin{array}{cc}
f_{i, R e} I_{T} & -f_{i, I m} I_{T} \\
f_{i, I m} I_{T} & f_{i, R e} I_{T}
\end{array}\right],}
\end{array}
$$

is the equivalent channel matrix and the $2 T \times 1$ real vector,

$$
\begin{array}{r}
\mathcal{W}=\left[\begin{array}{c}
\mathbf{w}_{R e} \\
\mathbf{w}_{I m}
\end{array}\right]+\sqrt{\frac{P_{2}}{P_{1}+1}} \sum_{i=1}^{R}\left[\begin{array}{cc}
g_{i, R e} I_{T} & -g_{i, I m} I_{T} \\
g_{i, I m} I_{T} & g_{i, R_{e} I_{T}}
\end{array}\right] \\
{\left[\begin{array}{cc}
A_{i}+B_{i} & 0 \\
0 & A_{i}-B_{i}
\end{array}\right]\left[\begin{array}{c}
\mathbf{v}_{i, R e} \\
\mathbf{v}_{i, I m}
\end{array}\right],}
\end{array}
$$

is the equivalent noise. Since this system equation is linear in the unknowns, which are entries of $\mathbf{x}$, sphere decoding can be used.

Using the optimum power allocation in (8), the PEP of mistaking $\mathrm{s}_{i}$ by $\mathrm{s}_{j}$ has the following Chernoff upper bound for large $P$.

$$
P e \leq \underset{g_{i}}{\mathrm{E}} \operatorname{det}^{-1 / 2}\left(I_{2 R}+\frac{P T}{8\left(R+\sum_{k=1}^{R}\left|g_{k}\right|^{2}\right)} \sum_{k=1}^{R} \mathcal{G}_{k} \mathcal{G}_{k}^{t}\right),
$$

where

$$
\begin{aligned}
\mathcal{G}_{k}=\left[\begin{array}{cc}
g_{k, R e} I_{T} & -g_{k, I m} I_{T} \\
g_{k, I m} I_{T} & g_{k, R e} I_{T}
\end{array}\right]\left[\begin{array}{cc}
A_{k}+B_{k} & 0 \\
0 & A_{k}-B_{k}
\end{array}\right] \\
\\
{\left[\begin{array}{ll}
\left(\mathbf{s}_{i}-\mathbf{s}_{j}\right)_{R e} & -\left(\mathbf{s}_{i}-\mathbf{s}_{j}\right)_{I m} \\
\left(\mathbf{s}_{i}-\mathbf{s}_{j}\right)_{I m} & \left(\mathbf{s}_{i}-\mathbf{s}_{j}\right)_{R e}
\end{array}\right] . }
\end{aligned}
$$

We have not yet been able to explicitly evaluate the expectation in (19). Our conjecture is that when $T \geq R$, the same transmit diversity, $R\left(1-\frac{\log \log P}{\log P}\right)$, will be obtained. Here we give an analysis of a much simpler, but far from trivial, case: for any $i$, either $A_{i}=0$ or $B_{i}=0$. That is, each relay node sends a signal that is either linear in its received signal or linear in the conjugate of its received signal. It is clear to see that Alamouti's scheme is included in this case with $R=2, A_{1}=I_{2}, B_{1}=0, A_{2}=0$, and $B_{2}=\left[\begin{array}{ll}0 & 1 \\ 1 & 0\end{array}\right]$. The conditions that $A_{i}+B_{i}$ and $A_{i}-B_{i}$ are orthogonal become that $A_{i}$ is orthogonal if $B_{i}=0$ and $B_{i}$ is orthogonal if $A_{i}=0$.

Theorem 5. Design the transmit signal at the ith relay node as in (17). Use the optimum power allocation in (8). Further assume that for any $i=1, \cdots, R$, either $A_{i}=0$ or $B_{i}=0$. The PEP of mistaking $\mathbf{s}_{i}$ by $\mathbf{s}_{j}$ has the following Chernoff upper bound.

$$
P e \leq \underset{g_{i}}{\mathrm{E}} \operatorname{det}^{-1}\left[I_{R}+\frac{P T}{8\left(R+\sum_{i=1}^{R}\left|g_{i}\right|^{2}\right)} \hat{M} G\right],
$$

where

with

$$
\hat{M}=\left(\hat{S}_{i}-\hat{S}_{j}\right)^{*}\left(\hat{S}_{i}-\hat{S}_{j}\right)
$$

$$
\hat{S}_{i}=\left[A_{1} \mathbf{s}_{i}+B_{1} \overline{\mathbf{s}_{i}}, \cdots, A_{R} \mathbf{s}_{i}+B_{R} \overline{\mathbf{s}_{i}}\right]
$$

$a T \times R$ matrix which is the distributed space-time code.

(20) is exactly the same as (9) except that now the distributed space-time code is $\hat{S}$ instead of $S$. Therefore, exact the same transmit diversity is obtained as in Sections V and VI. The coding gain for very large $P(P \gg \log P)$ is $\operatorname{det} \hat{M}$. When $P$ is not very large, we want not only $\operatorname{det} \hat{M}$ to be large but also all $\operatorname{det}\left[\left.\hat{M}\right|_{i_{1}, \ldots, i_{k}}\right.$ to be large. That is, to have good performance for not very large transmit power, the distributed space-time code should have the property that it is "scale-free" in the sense that it is still a good distributed spacetime code when some of the relays are not working.

\section{SimUlation RESULTS}

In this section, the performance of LD codes implemented distributively over wireless relay networks is compared with that of the same codes in multiple-antenna systems. Since the actual design of LD codes and their optimality is not an issue, here $A_{i}$ are generated randomly based on the isotropic distribution. The transmit signals at relays are designed as in (2). $s_{t}$ are designed as independent $N^{2}$-QAM signals. The rate of the code is therefore $2 \log N$.

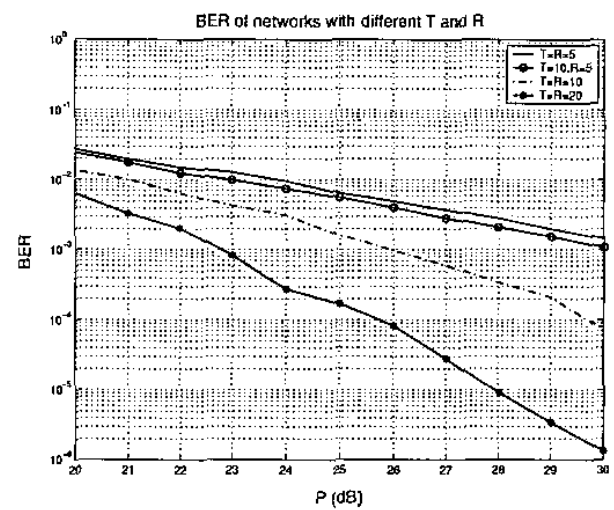

Figure 2: BER comparison of networks at different $T$ and $R$

In Fig. 2, we compare the bit error rate (BER) of relay networks with different coherence interval $T$ and number of relay nodes $R$. From the plot we can see that the bigger $R$, the faster the BER curve decreases, which verifies our analysis that the diversity is linear in $R$ when $T \geq R$. However, the slopes of the BER curves of networks with $T=R=5$ and $T=10, R=5$ are the same. This verify our result that the diversity only depends on $\min \{T, R\}$, which is always $R$ in our examples. Increasing $T$ does not improve the diversity.

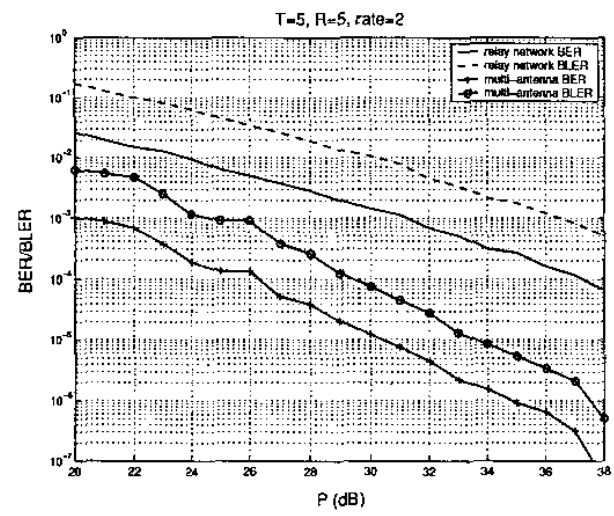

Figure 3: Comparison of the relay network with the multipleantenna system with $T=R=5$ and rate $=2$

In Fig. 3, the performance of a relay network with $T=R=5$ is compared with a multiple-antenna system with five transmit antennas and one receive antenna using the same LD code. $N=2$. From the figure we can see that the performance of the multiple-antenna system is always better than of the relay network at any $P$. This is what we expected because in the multiple-antenna system, the transmit antennas of the transmitter can fully cooperate and have perfect 
information of the transmit signal. Also we can see from Fig. 3 that the BER and BLER (block error rate) of the multiple-antenna system decrease faster than those of the relay network. However, the differences of the slopes of the curves of the two systems are diminishing as $P$ increases. Also, at the BER of $10^{-4}$, the total transmit power of the relay network is about $37.5 \mathrm{~dB}$. Our analysis of (14) indicates that the performance of the relay network should be $12.36 \mathrm{~dB}$ worse. Reading from the plot, we get a $11.5 \mathrm{~dB}$ difference. This verifies the correctness and tightness of our upper bound.

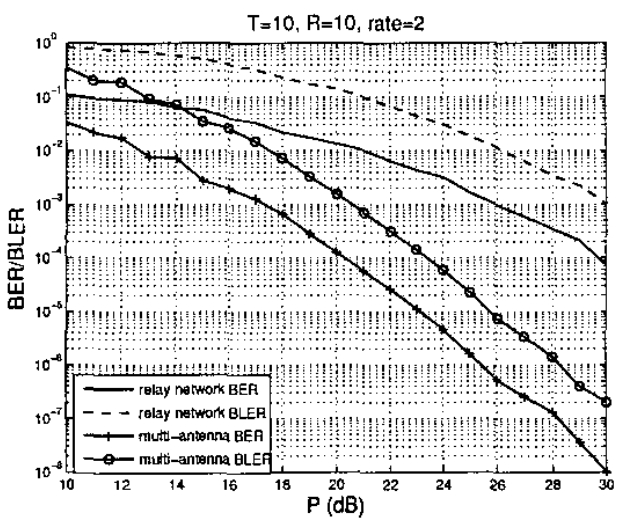

Figure 4: Comparison of the relay network with the multipleantenna system with $T=R=10$ and rate $=2$

The next example has $T=R=10$ and $N=2$. From Fig. 4 , the same phenomenon can be observed.

\section{CONCLUSION}

In this paper, the use of linear dispersion space-time codes in wireless relay networks is proposed. We assume that the transmitter and relay nodes do not know the channel realizations but only their statistical distribution. The ML decoding and pairwise error probability at the receiver is analyzed. The main result is that the diversity of the system behaves as $\min \{T, R\}\left(1-\frac{\log \log P}{\log P}\right)$, which shows that when $T \geq R$ and the average total transmit power is very high $(P \gg \log P)$, the relay network has almost the same diversity as a multiple-antenna system with $R$ transmit antennas and one receive antenna. It is further shown that, assuming $R=T$, the leading order

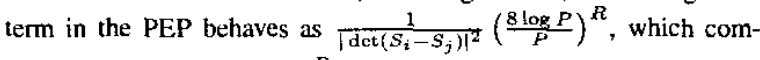
pared to $\frac{1}{\left|\operatorname{det}\left(S_{i}-S_{j}\right)\right|^{2}}\left(\frac{4}{P}\right)^{R}$, the PEP of a space-time code, shows the loss of performance due to the fact that the code is implemented distributively and the relay nodes have no knowledge of the transmitted symbols. We also observe that the high SNR coding gain, $\left|\operatorname{det}\left(S_{i}-S_{j}\right)\right|^{-2}$, is the same as that arises in space-time coding. The same is true at low SNR where a trace condition comes up.

We then improve the achieved diversity gain slightly (by no more than $O\left(\frac{\log \log P}{\log ^{2} P}\right)$ ). Furthermore, a more general type of distributed space-time linear codes is discussed, in which the transmission signal from each relay node to the receive node is designed as a linear combination of both its received signal and the conjugate of its received signal. For a special case, which includes the Alamouti's scheme, exactly the same diversity gain can be obtained. Simulation results on some randomly generated distributed space-time codes are demonstrated, which verify our theoretical analysis on both the diversity and coding gain.

\section{REFERENCES}

[1] I. E. Telatar, "Capacity of multi-antenna gaussian channels," Eur. Trans. Telecom., vol. 10, pp. 585-595, Nov. 1999.

[2] T. L. Marzetta and B. M. Hochwald, "Capacity of a mobile multipleantenna communication link in Rayleigh flat fading." IEEE Trans. Info. Theory, vol. 45, pp. 139-157, Jan. 1999.

[3] G. J. Foschini, "Layered space-time architecture for wireless communication in a fading environment when using multi-element antennas," Bell Lubs. Tech. J., vol. 1, no. 2, pp. 41-59, 1996.

[4] V. Tarokh, N. Seshadri, and A. R. Calderbank, "Space-time codes for high data rate wireless communication: Performance criterion and code construction," IEEE Trans. Info. Theory, vol, 44, pp. 744-765, 1998.

[5] A. Sendonaris, E. Erkip, and B. Aazhang, "User cooperation diversitypart I: System description," IEEE Transactions on Communications, vol. 51, pp. 1927-1938, Nov. 2003.

[6] A. Sendonaris, E. Erkip, and B. Aazhang, "User cooperation diversitypart II: Implementation aspects and performance analysis," IEEE Transactions on Communications, vol. 51, pp. 1939-1948, Nov. 2003.

[7] Y. Tang and M. C. Valenti, "Coded transmit macrodiversity: block spacetime codes over distributed antennas," in Vehicular Technology Conference. 2001, vol. 2, pp. 1435 - 1438, May 2001.

[8] J. N. Laneman and G. W. Wornell, "Distributed space-time-coded protocols for exploiting cooperative diversity in wireless betwork," IEEE Truns. on Info. Theory, vol. 49, pp. 2415-2425, Oct. 2003.

[9] R. U. Nabar, H. Bolcskei, and F. W. Kneubuhler, "Fading relay channels: Performance limits and space-time signal design," IEEE Joumal on Selected Areas in Communications, 2004, to appear.

[10] Y. Hua, Y. Mei, and Y. Chang, "Wireless antennas-making wireless communications perform like wireline communications," in IEEE AP-S Topical Conference on Wireless Communication Technology, Oct. 2003.

[11] Y. Chang and Y. Hua, "Application of space-time linear block codes to parallel wiretess relays in mobile ad hoc networks," in the Thirty-Sixth Asilomar Conference on Signals. Systems and Computers, Nov. 2003.

[12] B. Hassibi and B. Hochwald, "High-rate codes that are linear in space and time," IEEE Trans. Info. Theory, vol. 48, pp. 1804-1824, July 2002.

[13] A. F. Dana, M. Sharif, R. Gowaikar, B. Hassibi, and M. Effros, "Is broadcast plus multi-access optimal for gaussian wireless network?", in the 36th Asilomar Conf. on Signals, Systems and Computers, Nov, 2003.

[14] M. Gasptar and M. Vetterli, "On the capacity of wireless networks: the relay case," in IEEE Infocom, June 2002.

[15] A. F. Dana and B. Hassibi, "On the power-efficiency of sensory and adhoc wireless networks," Submitted to IEEE Trans. Info. Theory, 2003.

[16] Y. Jing and B. Hassibi, "Distributed space-time coding in wireless relay networks-part 1: Basic diversity results," Stobmitted to IEEE Transactions on Wireless Communications, 2004.

[17] Y. Jing and B. Hassibi, "Distributed space-time coding in wireless relay networks-part II: Tighter bounds and a more general case," Submitted to IEEE Transactions on Wireless Communications, 2004.

[18] M. O. Damen, K. Abed-Meraim, and M. S. Lemdani, "Further results on the sphere decoder algorithm," Submitted to IEEE Trans. Info. Theory, 2000.

[19] B. Hassibi and H. Vikalo, "On the expected complexity of integer leastsquares problems," in Acoustics, Speech, and Signal Processing, 2002 IEEE International Conference, April 2002.

[20] B. M. Hochwald and T. L. Marzetta, "Unitary space-time modulation for multiple-antenna communication in Rayleigh flat-fading," IEEE Trans. Info. Theory, vol. 46, pp. 543-564, Mar. 2000.

[21] M. Evans, N. Hastings, and B. Peacock, Statistical Distributions. Wiley, 2nd ed., 1993.

[22] I. S. Gradshteyn and I. M. Ryzhik, Table of Integrals. Series and Products. Academic Press, 6nd ed., 2000.

[23] H. L. V. Trees, Detection, Estimation, and Modulation Thenry-Part 1. New York: Wiley, 1968. 